\title{
Who's paying for new drugs?
}

Robert K. Oldham

Direct payment by patients for treatment with drugs still in development is the subject of controversy. The matter goes deeper than it might first appear.

Drug development is expensive and slow. Each new anti-cancer drug, for example, comes to the practising oncologist in the United States at a cost of $\$ 60-$ $\$ 100$ million and after a period of $8-12$ years of research. The major portion of costs and time is taken up by clinical trials designed to demonstrate the drug's effectiveness and acceptable toxicity to federal regulatory authorities.

The classic pattern of drug development requires thorough studies in vitro and in animal models, followed by Phase I clinical studies on human beings to delineate toxicities. Phase II studies are then conducted to determine the drug's efficacy. Given acceptable toxicity and some evidence of anti-cancer activity, Phase III trials are carried out to demonstrate a benefit in patient survival or quality of life, and to compare the new drug with existing treatments. This has been the process of drug development for over 30 years, and it is uniformly applied to anti-cancer (and most other) drugs by the Food and Drug Administration (FDA), pharmaceutical companies, and government and university researchers.

Over the past three years, my own company has been criticized for accepting patients' money for laboratory research services leading to experimental treatments. But it is pertinent to ask who, elsewhere in the clinical research system, is footing the bill for trials with new drugs.

\section{Grants and contracts}

Historically, pharmaceutical companies and the government awarded grants and contracts to universities for the conduct of clinical trials. The financial support for these trials came from profits accumulated from the sale of pharmaceutical products (pharmaceutical companies) and funds from taxpayers (government-sponsored trials). Obviously, the former funds originally belonged to patients and the latter were paid by patients and taxpayers. But hitherto a direct link between the patient and the funding of clinical trials research has not been apparent.

As the National Cancer Institute (NCI) has built up its network of Cancer Centers, Community Hospital Oncology Programs and Community Clinical Oncology Programs, however, the connection between drug development and patients paying for research has become more obvious. Phase I and II trials are still largely conducted by universities and, in theory, the funding for these trials comes from pharmaceutical company grants and contracts, as well as taxpayers' dollars. But even for these early trials (for example of interleukin-2/activated killer cells), there has recently been a closer linkage with patient funding. Patients and their insurance companies are being billed for hospital and laboratory costs, and sometimes for professional fees. These clinical research protocols are uniformly peer reviewed and approved with the investigational agents provided by the NCI and/or the sponsoring pharmaceutical company. All require FDA registration, because the research is being carried out under an investigational new drug (IND) application cleared by the FDA.

It is clear that insurance companies are increasingly being charged for such trials through standard hospital and clinic billing systems. There has been a great deal of debate on the Diagnosis Related Group (DRG) system as it relates to research funding. The lack of a research DRG has made more apparent the lack of access to experimental medicine of those depending on Medicare. Private insurance companies continue to be billed for clinical research, however, sometimes without the insurance company being informed that the patient was being treated under an experimental protocol.

Phase III trials are even more closely connected to the practice of medicine and the standard method of insurance reimbursement in the United States. For experimental trials of anti-cancer drugs, the protocols are often conducted in cancer centres and as part of NCI clinical cooperative groups. These cancer centres and cooperative groups derive a large portion of their funding from the NCI and their protocols are reviewed and approved within the NCI system.

The Phase III protocols often involve testing two chemotherapeutic drugs against three others, or one sequence of administration against another. The drugs may be investigational, with testing under an IND, or they may be new combinations of approved drugs being used experimentally in sequences or combinations not yet approved for routine use. Because drugs are approved by the FDA for other indications, and because the studies of toxicity and efficacy are often based on trials conducted with the drug as a single agent, new combinations and sequences represent the experimental use of drugs even when the agents are commercially available.

\section{Experimental protocols}

Theoretically, these combinations and sequences should have Phase I (toxicity) and Phase II (efficacy) testing before they are put into widespread use. But this has not been the case. Physicians, pharmaceutical companies and the NCI routinely conduct Phase III trials of new combinations and novel sequences as experimental protocols; these protocols are carried out as clinical research and published in medical journals. Although there is some taxpayer support for such trials through NCI cancer centres and cooperative group programmes, the bulk of the hospital, clinic and laboratory costs is paid for by insurance companies and the patients. Insurance payment for experimental medicine represents patient-funded research, and the difference between the insurance reimbursement and the billed amount is often paid by the patient. Thus, the main source of funding for Phase III trials such as these is the patient.

When costs are paid directly by the patient, the patient-funded research linkage is clear for all to see. When an insurance premium is paid and insurance reimbursement covers these costs, it is still patient-funded research with a somewhat less clear linkage. Similarly, when a pharmaceutical company uses research and development funds derived from the sale of approved drugs to pay for research trials with new products, the money actually comes from patients paying for approved products and is thus patientfunded research. This is yesterday's patient paying for tomorrow's patient's research. The linkage is here one step further removed from obvious patientfunded research. Finally, when taxpayer dollars are used for research, the funding is coming from a mixture of individuals most of whom are not yet patients.

Is it acceptable for patients to fund research indirectly but not acceptable when the direct relationship is apparent? The question of who pays for clinical research needs broad public debate and openness on the part of all the parties involved in developmental therapeutics.

Robert K. Oldham is Director of the Biological Therapy Institute, Hospital Drive, Franklin, Tennessee 37064, USA. 